\title{
Complications of mediastinoscopy
}

\author{
Laura Socci' ${ }^{1}$, Sara Sionis ${ }^{2}$, Annabel Sharkey ${ }^{1}$ \\ ${ }^{1}$ Cardiothoracic Department, Sheffield Teaching Hospitals, Sheffield, UK; ${ }^{2}$ Ear Nose Throat Department, Sheffield Teaching Hospitals, Sheffield, UK \\ Contributions: (I) Conception and design: L Socci; (II) Administrative support: None; (III) Provision of study materials or patients: None; (IV) \\ Collection and assembly of data: None; (V) Data analysis and interpretation: None; (VI) Manuscript writing: All authors; (VII) Final approval of \\ manuscript: All authors. \\ Correspondence to: Laura Socci. Thoracic Surgeon, Sheffield Teaching Hospitals, Sheffield, UK. Email: laura.socci@nhs.net.
}

\begin{abstract}
The most common mediastinoscopy technique is the video-assisted cervical mediastinoscopy (VAMLA) which is the modern version of the original mediastinoscopy described by Carlens in 1959. The extended-VAMLA and the trans-cervical extended mediastinal lymphadenectomy (TEMLA) techniques permit to biopsy a wider number of lymphonodal stations but they do carry out a high operative risk and are not widespread utilised. The most common perioperative complications in relation to a mediastinoscopy described in literature have been divided in 6 categories: (I) bleeding; (II) left laryngeal nerve palsy; (III) infection; (IV) pneumothorax; (V) tracheal perforation; (VI) oesophageal perforation. Of those, the first three categories are generally considered the most common ones. Of each category we describe tips to try to avoid the complication and the most common surgical management of the complication as presented in literature. In two categories as well, we present a patient's case from our unit experience including related imaging. The mediastinoscopy is nowadays a routine procedure for a Thoracic Unit and commonly is managed as a day case procedure. Despite being considered a minor procedure it does need a specific training and carries out specific risks, which can vary from minor to catastrophic. The use of a video system permits a safe training and a safer and procedure.
\end{abstract}

Keywords: Mediastinoscopy; complication; iatrogenic

Received: 29 July 2021; Accepted: 10 August 2021; Published: 30 October 2021.

doi: $10.21037 /$ shc-21-20

View this article at: https://dx.doi.org/10.21037/shc-21-20

\section{Introduction}

The cervical mediastinoscopy technique has firstly been described by Dr. Carlens in 1959 (1-4).

Nowadays a video-assisted cervical mediastinoscopy (VAMLA) is the gold standard modern version of this technique: the mediastinoscope is connected to an HD (high definition) video system, which magnifies the structures and increase the safety of the dissection. It is important to note that while with the Carlens version only the operator does have view and access to the operating field, with the VAMLA the assistant and the team can follow the procedure on the monitor, obviously increasing the awareness and learning of the team and the safety itself of the operation.

It is as well the recommended technique for training purpose for the above reasons.
Patient's selection is crucial.

Relative contraindications are: previous tracheostomy, neck oncological dissection or previous neck irradiation as part of oncological treatment, mediastinoscopy, cervical spine disorders, cerebral vascular disorders, coagulopathies.

Absolute contraindications: impossibility to extend the neck (severe kyphosis), tracheal stoma due to laryngectomy.

The position of the patient is paramount to obtain a good exposure and to minimise surgical risks. The patient will be positioned supine with an inflatable bag carefully placed underneath the shoulders, in this way the neck of the patient is well extended, the endotracheal tube is positioned in one of the sides of the mouth and not centrally.

A $3 \mathrm{~cm}$ curvilinear incision is made $1 \mathrm{~cm}$ above the sternal notch; the pretracheal layers are dissected as per a 
conventional tracheostomy. The tracheal fascia is entered and this manoeuvre consent the surgeon to introduce safely the mediastinoscope below the pretracheal fascia into the superior/middle mediastinum.

Surgeon and assistant usually are positioned to the head side of the operating table.

With a conventional mediastinoscopy the superior and inferior paratracheal lymphonodes, and subcarinal lymphonodes are commonly accessible (stations $2 \mathrm{R}-2 \mathrm{~L}$, 4R-4L, 7).

The instruments utilised are a combined suction-cautery device and a biopsy forceps. A bimanual technique is performed: while with the forceps the lymphonode is gently pulled and twisted with the left hand, with the right hand the suction-cautery device allows a careful dissection and cauterization of small lymphatic vessels.

Indications: the technique is commonly used for diagnosis and staging of non-small cell lung cancer (NSCLC), lymphoma, sarcoidosis, infective disorders (tuberculosis most frequently), and rarely parathyroid adenoma $(2,3)$.

Regarding the NSCLC it is important to note that when the mediastinal lymphonodes are enlarged and suitable for endobronchial ultrasound guided biopsy (EBUS) biopsy this method should be the preferred pre-treatment one, whilst the VAMLA should be reserved as a post-induction treatment restaging technique. It is not recommended to perform a redo VAMLA as the procedure carries higher risk of intraoperative complications.

In the current practise the procedure is commonly offered as day case minimising the hospital stay only up to few hours after the procedure.

From the literature VAMLA shows a very low risk of mortality $(0.09 \%)$, morbidity $(0.83-2.9 \%)$, and accuracy (87.9-98.9\%) (3).

The extended cervical mediastinoscopy is the modified technique introduced by Dr. Kirschner in 1971. This version of the technique allows the biopsy of the aortopulmonary window (station 5) and para-aortic region (station 6) passing the mediastinoscope around the innominate vein $(5,6)$.

These regions are easily and safely biopsiable with a left video-assisted thoracoscopic surgery (VATS) technique too which minimises the risk of complications.

TEMLA, or trans-cervical extended mediastinal lymphadenectomy, is an even more aggressive version of the technique which again is currently offered only in few centres worldwide. TEMLA is performed through a 5- to
8 -cm collar incision in the neck and enables the complete removal of all mediastinal nodal stations except for the pulmonary ligament nodes (station 9) (6).

To resume the most common complications in relation with VAMLA that are described in literature and that we are going to discuss are:

(I) Bleeding;

(II) Left laryngeal nerve palsy;

(III) Infection;

(IV) Pneumothorax;

(V) Tracheal perforation;

(VI) Oesophageal perforation.

\section{Bleeding}

Bleeding during mediastinoscopy can vary between small and well controllable to disastrous and fatal.

The surgeon should apply a careful haemostasis prior closing the wound. The common bleeding sites are lymphonodal bed and lymphonodal vessels, which can be cauterized with an insulated diathermy sucker. It is very important to avoid pulling hardly the lymphonodes from the vascular structures; it is wiser to carefully dissect the lymphonode from the vessel.

Because the working space is very small even a minimal bleeding can cause the total loss of visualization. In case of bleeding, it is recommended to do not remove the mediastinoscope but to use suction to aspirate the blood whilst the lens can be cleaned instilling a small amount of water through the working channel of the videomediastinoscope, a swab can be inserted through the instrument for packing purpose and should be kept in place for few minutes.

In case of minor bleeding and especially on the left paratracheal region a surgical haemostatic agent can be utilised in order to avoid the usage of cautery in that region which can lead to left laryngeal nerve palsy.

In case of a major bleeding (from a major vessel) it is mandatory to pack the mediastinum inserting a surgical swab into the mediastinoscope and carefully retrieving the instrument while applying pressure from the chest leaving the swab in place. The surgical bed can be raised to a $30^{\circ}$ reverse-Trendelenburg position while applying packing compression. The operating team needs to be prepared to deal with a massive haemorrhage. A sternotomy or a thoracotomy approach will give access to direct repair of the vessel injury, a major bleeding transfusion pack should be ordered. 


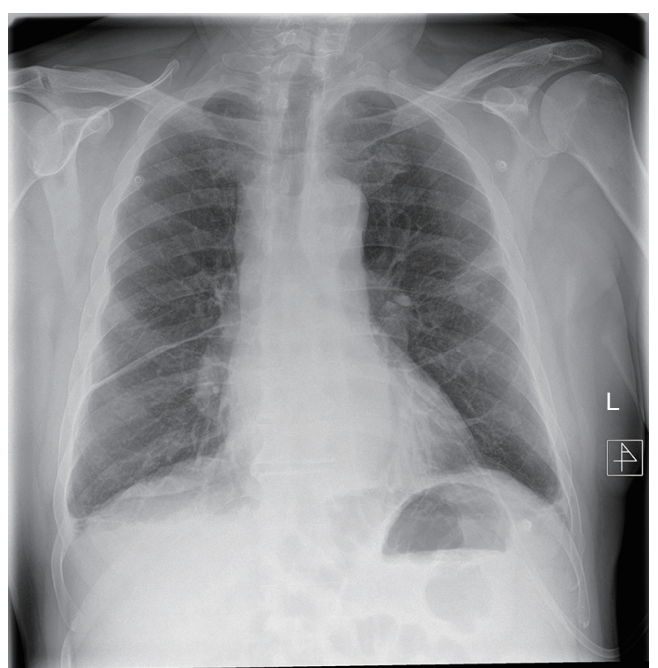

Figure 1 Chest $\mathrm{X}$-ray of the patient at re-admission showing enlarged mediastinal silhouette.

We describe a rare case of a postoperative mediastinal bleeding that occurred in our service related to coagulopathy disorder (Figures 1,2).

This 66-year-old man initially presented with haemoptysis and was found to have a left upper lobe lesion on computed tomography (CT). Positron emission tomography (PET) scan revealed a nodule with an SUVmax of 6.9 in the left upper lobe and hilar and paratracheal lymph nodes with SUVmax of 4.1 and 3.1 respectively, all suspicious for primary lung cancer. He was referred for mediastinoscopy for staging and diagnosis purposes. Past medical history included being an ex-smoker, myelofibrosis on medical treatment, chronic obstructive pulmonary disease (COPD), hypertension and gastrooesophageal reflux. Preoperative haematological advice was to continue his medications. He underwent a cervical videomediastinoscopy, which was routine and uneventful. Stations 2R, 2L, 4R, 4L and 7 were sampled. There was minimal expected bleeding experienced when the biopsies were taken and haemostasis was achieved prior to closure. He was discharged home the same day.

Later that evening he re-presented with upper abdominal pain. Investigations revealed abnormal clotting with a slightly raised partial thromboplastin time (PTT) and activated partial thromboplastin time (aPTT), a raised white cell count and haemoglobin that had decreased by $10 \mathrm{~g} / \mathrm{L}$ from pre-operatively. Chest X-ray showed a slightly widened mediastinum. A CT scan showed a mediastinal haematoma predominantly in the subcarinal region, extending inferiorly to the oesophageal hiatus and superiorly around the carina and distal trachea, along with bilateral pleural effusions, right larger than left. Aortic and bronchial angiogram did not demonstrate a bleeding point, and also found that anyway the bronchial arteries were very small and not easily amenable to embolization. Haematological advice this time supported the impression of coagulopathy due to myelofibrosis, replacement of coagulation factors was prescribed.

Due to the concern of persistent small bleeding the patient underwent a right VATS drainage of haemothorax and exploration of the mediastinum. The mediastinal pleura was opened fully and drained but no specific bleeding point could be identified. The subcarinal region was explored round to the origin of the left main bronchus where there was possibly a bleeding point from the bronchial artery. Haemostasis was achieved and the chest washed out with saline and $3 \mathrm{~g}$ tranexamic acid. He then underwent a left VATS evacuation of haemothorax, the subcarinal region was explored and no active bleeding point was identified from the left bronchial artery. He finally made a steady recovery and was eventually safely discharged.

\section{Left laryngeal nerve palsy}

Vocal cord paralysis (VCP) is a well-known complication of thoracic surgery (7-11).

The recurrent laryngeal nerve (RLN), also known as the inferior laryngeal nerve, is a branch of the vagus nerve which typically has a characteristic loop around the subclavian artery on the right and the aortic arch on the left, before returning up to run in the tracheoesophageal groove and then innervate the larynx.

The left RLN, with its longer intrathoracic segment, is more vulnerable to injury during thymic, mediastinal, tracheal and lung surgery. For this reason, a VCP after thoracic surgery is almost always left-sided, due to the surgical section, traction or heat-related injury of the vagus nerve or RLN during the lymphonodal dissection on the left paratracheal and aorto-pulmonary window spaces. While dissecting lymphonodes in this area extreme care should be applied by the surgeon to avoid palsy of the nerve. In particular the surgeon should avoid or limit the usage of cautery, which anyway should be used in a low intensity modality.

The RLN innervates all the intrinsic laryngeal muscles via its anterior and posterior branches, apart from the cricothyroid muscle which is supplied by the superior 

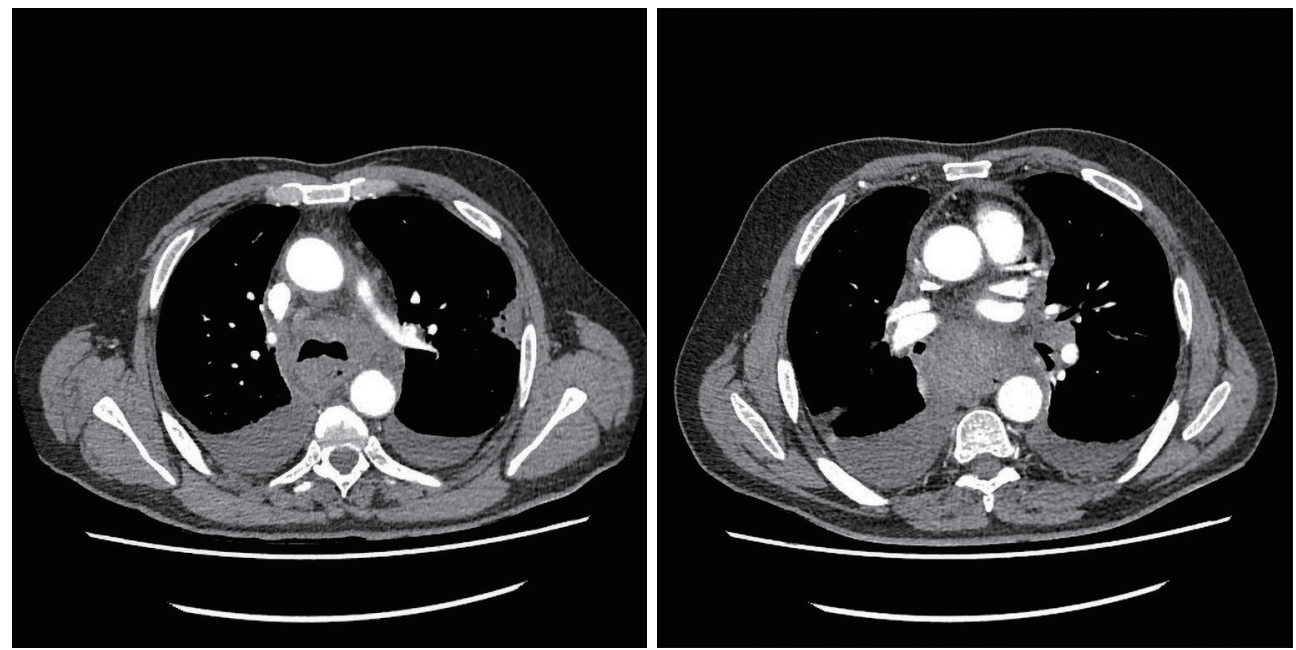

Figure 2 CT scan of the patient at re-admission confirming mediastinal bleeding and bilateral pleural effusions. No active bleeding point was possible to confirm radiologically. CT, computed tomography.

laryngeal nerve (SLN). Innervation of the interarytenoid muscle is classically described as bilateral, via the RLN and SLN (12).

Unilateral injury of the RLN results in paralysis of all the ipsilateral intrinsic laryngeal muscles with the exception of the interarytenoid and cricothyroid muscles.

The vocal cord has been described as assuming a paramedian position, although it is not uncommon for the vocal cord to lie in median or more lateral position.

In instances of complete vagal section, the vocal cords have been reported to assume an intermediate (cadaveric) position. In such instance, the cords are more lateral than with isolated RLN injury due to loss of adduction otherwise provided by the cricothyroid muscle when the SLN is intact.

Some studies have shown that immediately following sectioning of SLN and RLN on the same side, the vocal cord initially assumes a paramedian position at rest and then lateralises progressively due to the long-term effects of a sustained paralysis. This may, however, differ due to compensation from the other laryngeal and neck muscles. (13,14).

Depending on the type and site of injury, the VCP can therefore lead to dysphonia or aphonia with or without aspiration, or respiratory distress.

Urgent referral to the Otolaryngologist should be done in patients with symptoms of VCP following surgery, for immediate diagnosis and therapeutic strategy to minimise postoperative complications and to overcome any impairment of the voice and swallowing function.

Clinically, vocal cord function is assessed by laryngoscopy, during which the Otolaryngologist can confirm a paralysis in abduction or adduction.

In case of unilateral VCP in abduction (paramedian, intermediate or lateral position), the patient will present with various grades of dysphonia (hoarseness, vocal fatigue, loss of vocal pitch) frequently associated with cough and dysphagia with or without aspiration.

Speech and language therapy assessment and vocal cord medialisation techniques such as injection laryngoplasty and medialisation thyroplasty should be considered.

In the case of unilateral VCP in adduction (median position), the patient will present with shortness of breath due to the reduced airway. Lateralisation laryngoplasty and endoscopic posterior cordotomy are the treatment of choice in selected cases.

Emergency tracheostomy is usually required for patients with bilateral vocal cord palsy in adduction to secure the airway.

\section{Infection}

The risk of wound infection is rare after applying the common surgical skin preparation.

Nevertheless, the biopsy of mediastinal lymphonodes, which may be affected by an infective disorder, could be the carrier of mediastinal space infection in case of spillage during the procedure. Such rare cases should not be 


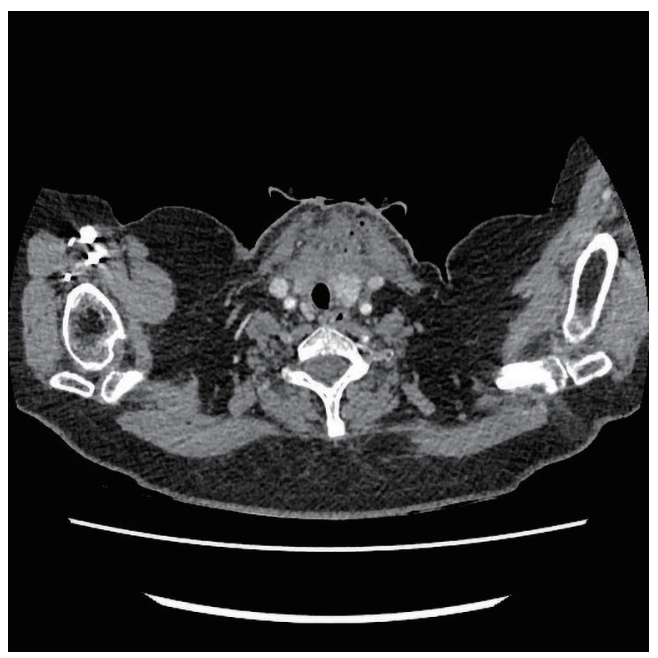

Figure 3 Wound and superior mediastinal collection with gas locules visible at the CT scan. CT, computed tomography.

underestimated and be treated as an acute mediastinitis. A CT scan will be a paramount test to identify the extent of the infection. The surgical approach to treat the infection can vary from a wound re-exploration with irrigation and drainage utilising the original surgical wound if the infection has been recognised early and the CT scan confirms only supero-anterior mediastinum collection, to a bilateral VATS mediastinal and chest cavities drainage.

Intravenous broad-spectrum antibiotics should be administered in first instance.

We present a case of early postoperative wound and superior mediastinum infection occurred in our unit.

This 52-year-old lady was referred for a diagnostic mediastinoscopy after routine CT follow up of previously treated breast cancer revealed new mediastinal lymphadenopathy. She underwent cervical videomediastinoscopy and biopsy of stations $2 \mathrm{~L}$ and $4 \mathrm{R}$ lymph nodes. Upon biopsy of 4R, white fluid was drained and this was sent for culture. The patient was discharged on the same day as per our practice. Two days later she was brought back to the hospital as her lymph node sample had grown Streptococcus oralis and Streptococcus anginosus bacteria so she was started on oral ciprofloxacin (fluoroquinolone) accordingly to microbiology advice. On review a week post-surgery, at the end of the ciprofloxacin course, she was found to have a swollen fluctuant wound which was tender on palpation. A CT scan showed a collection containing gas loculi in the anterior neck measuring $50 \mathrm{~mm} \times 48 \mathrm{~mm}$ extending inferiorly into the superior mediastinum. She underwent re-exploration of the wound and washout of the mediastinum the following day. After a course of intravenous antibiotics, she was discharged home to continue a prolonged course of oral antibiotics (penicillin and clavulanic acid combination), she made a full recovery (Figure 3).

\section{Pneumothorax}

While dissecting carefully both of the paratracheal lymphonodal regions the pleura should be identified and a careful dissection of the lymphonode from the pleura and fat tissue surrounding it should be performed.

In case of a small tear of the pleura has been caused during this manoeuvre commonly it is not necessary to drain the pleural cavity, a Valsalva manoeuvre at the end of the procedure before closing the wound is considered sufficient.

A postoperative chest $\mathrm{X}$-ray performed within few hours from extubation can be useful in such cases.

The risk that a chest drain insertion is necessary in such cases is minimal.

\section{Tracheal perforation}

During the dissection especially of the anterior subcarinal lymphonodes the use of the cautery is necessary. It is imperative to be really careful on utilising the instrument in order to avoid damage of vessels or tracheal wall. In the rare event of a small anterior tracheal wall tear firstly it is important to assess the extent of the injury caused.

This can be done passing a flexible bronchoscope through the endotracheal tube while keeping the videomediastinoscope in place. With the bronchoscope is possible to assess the tracheal tear from the endotracheal view and verify the extent. Another manoeuvre that can be used is utilising the videomediastinoscope working channel: a small amount of water can be poured through the working channel and while the anaesthetist is perform a sustained inflation it is possible to assess the amount of bubbling produced (this is basically a water seal test through the videomediastinoscope).

For minimal and minor tracheal injuries, a conservative management is recommended, a sound wound closure is considered sufficient, a small drain can be left in place (15).

For moderate injuries a primary repair should be considered. The surgical approach depends on the level of the injury.

For high tracheal tears as the one caused during 
mediastinoscopy a traditional surgical approach can be utilised. An interesting minimally invasive approach of endoscopic tracheal suture has been proposed by Lancelin in 2000 (16), and recently published by da Silva Costa in 2012 (17), it is recommended only on expert hands.

\section{Oesophageal perforation}

Oesophageal perforation during a VAMLA is considered an extremely rare complication. Thus can occur while biopsying station $4 \mathrm{~L}$ and 7 if an aggressive lymphadenectomy is performed. It is imperative to try to avoid such injury.

In case of suspect of oesophageal perforation (acute postoperative chest pain and pneumo-mediastinum are pathognomonic) the patient should be fasted and a water contrast swallow or a CT scan with oral contrast will confirm the location and entity of the oesophageal tear. Broad-spectrum antibiotics and intravenous fluids should be initiated.

A direct repair of the tear from the lateral approach (either via VATS or thoracotomy) is recommended in cases of early diagnosis of iatrogenic oesophageal perforation, at the same time the chest cavity will be abundantly washed and then drained.

Alternatively, a conservative approach is based on the utilisation of an endo-oesophageal stent in order to exclude the perforation site and drainage of the infected spaces (mediastinum and chest cavities) (18).

\section{Conclusions}

The video mediastinoscopy technique is a common procedure utilised by thoracic surgeon for diagnostic and staging purpose.

It is in itself very safe and effective, of course requires specific training and instrumentation.

Nowadays most of the patients can be offered this procedure as a day case.

Unfortunately, there are specific risks of complications that must be discussed preoperatively with the patient and that need a clear intraoperative or postoperative strategy.

We have looked into the literature and into our unit casuistry and we describe and discuss the most frequent complications related to the procedure.

\section{Acknowledgments}

Funding: None.

\section{Footnote}

Provenance and Peer Review: This article was commissioned by the Guest Editor (David Waller) for the series "Complications of Thoracic Surgery - aetiology, management and prevention" published in Shanghai Chest. The article has undergone external peer review.

Conflicts of Interest: All authors have completed the ICMJE uniform disclosure form (available at https://dx.doi. org/10.21037/shc-21-20). The series "Complications of Thoracic Surgery - aetiology, management and prevention" was commissioned by the editorial office without any funding or sponsorship. All authors have no other conflicts of interest to declare.

Ethical Statement: All authors are accountable for all aspects of the work in ensuring that questions related to the accuracy or integrity of any part of the work are appropriately investigated and resolved.

Open Access Statement: This is an Open Access article distributed in accordance with the Creative Commons Attribution-NonCommercial-NoDerivs 4.0 International License (CC BY-NC-ND 4.0), which permits the noncommercial replication and distribution of the article with the strict proviso that no changes or edits are made and the original work is properly cited (including links to both the formal publication through the relevant DOI and the license). See: https://creativecommons.org/licenses/by-nc-nd/4.0/.

\section{References}

1. Carlens E. Mediastinoscopy: a method for inspection and tissue biopsy in the superior mediastinum. Dis Chest 1959;36:343-52.

2. Lerut T, De Leyn P, Coosemans W, et al. Cervical videomediastinoscopy. Thorac Surg Clin 2010;20:195-206.

3. De Leyn P, Lardinois D, Van Schil PE, et al. ESTS guidelines for preoperative lymph node staging for non-small cell lung cancer. Eur J Cardiothorac Surg 2007;32:1-8.

4. Leschber G, Sperling D, Klemm W, et al. Does videomediastinoscopy improve the results of conventional mediastinoscopy? Eur J Cardiothorac Surg 2008;33:289-93.

5. Ginsberg RJ, Rice TW, Goldberg M, et al. Extended cervical mediastinoscopy. A single staging procedure for bronchogenic carcinoma of the left upper lobe. J Thorac 
Cardiovasc Surg 1987;94:673-8.

6. Zieliński M. Transcervical extended mediastinal lymphadenectomy: results of staging in two hundred fiftysix patients with non-small cell lung cancer. J Thorac Oncol 2007;2:370-2.

7. Lodewyks CL, White CW, Bay G, et al. Vocal Cord Paralysis After Thoracic Aortic Surgery: Incidence and Impact on Clinical Outcomes. Ann Thorac Surg 2015;100:54-8.

8. Wright CD, Zeitels SM. Recurrent laryngeal nerve injuries after esophagectomy. Thorac Surg Clin 2006;16:23-33, v.

9. Ishimoto S, Ito K, Toyama M, et al. Vocal cord paralysis after surgery for thoracic aortic aneurysm. Chest 2002;121:1911-5.

10. Puccinelli C, Modzeski MC, Orbelo D, et al. Symptomatic unilateral vocal fold paralysis following cardiothoracic surgery. Am J Otolaryngol 2018;39:175-9.

11. Velez-Cubian FO, Toosi K, Glover J, et al. Transient Aphonia After Mediastinoscopy. Ann Thorac Surg 2017;103:e549-50.

12. Mu L, Sanders I, Wu BL, et al. The intramuscular innervation of the human interarytenoid muscle.

doi: $10.21037 /$ shc-21-20

Cite this article as: Socci L, Sionis S, Sharkey A. Complications of mediastinoscopy. Shanghai Chest 2021;5:43.
Laryngoscope 1994;104:33-9.

13. Syamal MN, Benninger MS. Vocal fold paresis: a review of clinical presentation, differential diagnosis, and prognostic indicators. Curr Opin Otolaryngol Head Neck Surg 2016;24:197-202.

14. Benninger MS, Crumley RL, Ford CN, et al. Evaluation and treatment of the unilateral paralyzed vocal fold. Otolaryngol Head Neck Surg 1994;111:497-508.

15. Denlinger CE, Veeramachaneni N, Krupnick AS, et al. Nonoperative management of large tracheal injuries. J Thorac Cardiovasc Surg 2008;136:782-3, 783.e1.

16. Lancelin C, Chapelier AR, Fadel E, et al. Transcervicaltranstracheal endoluminal repair of membranous tracheal disruptions. Ann Thorac Surg 2000;70:984-6.

17. da Silva Costa A Jr, Juliano Perfeito JA, Succi JE, et al. A video-assisted endotracheal suture technique for correction of distal tracheal laceration after intubation. Ann Thorac Surg 2012;93:2073-5.

18. Dasari BV, Neely D, Kennedy A, et al. The role of esophageal stents in the management of esophageal anastomotic leaks and benign esophageal perforations. Ann Surg 2014;259:852-60. 